\title{
Implementation and Integration of Fuzzy Algorithms for Descending Stair of KMEI Humanoid Robot
}

\author{
Wulandari Puspita Sari' ${ }^{1}$ R. Sanggar Dewanto ${ }^{2}$, Dadet Pramadihanto ${ }^{3}$ \\ 1Electrical Engineering Department, Politeknik Elektronika Negeri Surabaya \\ ${ }^{2}$ Mechanical Engineering Department, Politeknik Elektronika Negeri Surabaya \\ ${ }^{3}$ Computer Engineering Department, Politeknik Elektronika Negeri Surabaya \\ E-mail: ${ }^{1}$ wulandari.elektro28@gmail.com, ${ }^{2}$ sanggar@pens.ac.id, ${ }^{3}$ dadet@pens.ac.id
}

Received July 16, 2020; Revised September 2, 2020; Accepted October 30, 2020

\begin{abstract}
Locomotion of humanoid robot depends on the mechanical characteristic of the robot. Walking on descending stairs with integrated control systems for the humanoid robot is proposed. The analysis of trajectory for descending stairs is calculated by the constrains of step length stair using fuzzy algorithm. The established humanoid robot on dynamically balance on this matter of zero moment point has been pretended to be consisting of single support phase and double support phase. Walking transition from single support phase to double support phase is needed for a smooth transition cycle. To accomplish the problem, integrated motion and controller are divided into two conditions: motion working on offline planning and controller working online walking gait generation. To solve the defect during locomotion of the humanoid robot, it is directly controlled by the fuzzy logic controller. This paper verified the simulation and the experiment for descending stair of KMEI humanoid robot.
\end{abstract}

Keywords: descending stair, humanoid robot, fuzzy logic control, zero moment point.

\section{INTRODUCTION}

A humanoid robot is a robot that has a shape like a human. Design of humanoid robot can duplicate the human activity. The humanoid robot has two legs and two hands. The main factor of the stabilizer of the humanoid robot's whole bodies is the important thing. The robustness system in humanoid robots means that the robots perform tasks on several surfaces, such as normally walking on ground, stairs, and another surface. There are many issues of stable dynamic and gait trajectory walking on an uneven surface, such as the novel gait synthesis for stable walking.

The trajectories of feet are calculated by intelligent computing methods and by constraint analysis on stepping stairs [1]. Common concern reduces the errors of arising zero moment point effects on vertical and horizontal motions. Linear and angular accelerations are more important for the robot 
stability in dynamic walking [2]. Simplified model using an inverted pendulum has been introduced. There are two approaches for biped modeling: distributed mass model and real parameter simplified model. The detail information form of dynamic parameters of the robot is discussed in [3]. The robot generates 3D walking motions ascertains dynamically by using linear constraints with approach efficient MPC, and successfully during walking on uneven terrains also walking on the ground [4]. Scheme for trajectory planning walking on stairs constrains to parameter plane. The number of CoG and ZMP equations are limited for CoG motion [5]. Several trajectories are deployed in single and double stance [6]. The height of constant CoM is assumed in the single stance, while the cubic splines are used in the double support phase. Thus, it allows a transition to the different CoM heights. However, the reasons of using the approach method are not clear. Especially when demonstrating a stable walking on uneven terrain using simulations. A well-designed torso height has been applied for a selfstabilization to avoid kinematic constraints such as the joint limits [7]. Optimization frameworks have been used in [8] and [9] to generate the robot motions for long distance of walking by considering kinematic and dynamic constraints into the account.

Several environments can be changed for the stability of biped robot. Type of movement can be seen in trajectories. Parameter Constraint of walking on stairs is presented in trajectory planning [10]. Gait generation or footstep over stairs is obtained. Locomotion of biped robot can be calculated ideally through kinematics [11]. Strategy for bipedal walking generates trajectories including optimal gait trajectories from human walking data [12]. The trajectory of walking has two conditions: single support phase and double support phase. The system was calculated by retrieving ZMP of each foot for the DSP condition.

Many researchers built humanoid robots for climbing stairs using hybrid state-driven autonomous control in the condition of random perturbation, external force, and ununiform stairs. The robust system has been verified by the simulation of 12 DoF [13]. Gait planning for ascending and descending stairs of 7 DoF biped robot considers the power consumption and the balance of walking using GA and PSO algorithm [14]. Motion planner based on random algorithm is used to reach the sequence of footstep [15]. Trajectories of hip and ankle are built by inverted pendulum model verified on simulation [16]. Power consumption during walking up stairs needs more power than descending stairs [17]. The inverted pendulum model has been described in three dimensional with constraints the vertical motion by the shifting CoM [18]. Analytical approaches on the center of pressure through force distribution for optimized motion on uneven terrain are discussed [19].

In this research, a cubic polynomial approximation is used to design motions, and focus on vertical and horizontal force for optimal pattern generation. Strategy for walking on stair is applied by tracking CoP movements and CoM of the system. This point is valid even if the contact of 
the toe is located on support polygon. Walking pattern is generally divided into two conditions: first condition is SSP (Single Support Phase) condition of one leg swings, and the second condition is DSP (Double Support Phase) condition of two legs in the ground, considering stairs as virtual slopes. ZMP equation is used to calculate the distributed mass and considers the stability area. When Inverted pendulum is applied, ZMP equation can be calculated more precisely.

In real-time locomotion, the gait generation process cannot be used due to the consequences of using distributed mass of ZMP equation. In this paper, ZMP equation problems and ZMP definition problems are solved by using the virtual slope method. The detail information from the system dynamics is also used for the proposed trajectory generation method. Dynamic modeling section explains the specifications of the robot and the details of dynamic modeling, and gait planning section explains an overview of the inverted pendulum approach and the ZMP-based approach. Types of walking are built in the pattern generation which describes foot trajectory and hip trajectory that was an influence for walking downstairs. ZMP-based approach subsection describes the trajectory generation method for feet and pelvis, and the calculation of virtual SP and virtual ZMP.

Locomotion of humanoid robots is adopted from humans for natural conditions. When a robot dropped, it reflects on the human behavior. Gait generation for walking depends on the control strategy as feedforward controls and kinematics constraints. Sensory feedback is used to establish the control strategy or reflex behavior. Parameters of the feedback control are adjusted by fuzzy logic controller method. This research describes the analysis based on 33 DoF. We present a walking control strategy for humanoid robot descending stairs which consists of a novel gait descending stair and direct control using fusion sensors. Gait for descending stairs is divided into eight steps with full body control.

\section{RELATED WORKS}

Lin et al. [20] discuss fuzzy logic control, some of the researches are presented here. Developed control of fuzzy logic is an integrated modern theory of biped robot for dynamic stability and movement control. The experiment of the control brought nonlinear control and uncertain condition in characteristic of dynamic robot. However, they establish the membership function and the rule in fuzzy logic which cannot be modified.

Park et al. [21], fuzzy logic controller can be applied on several plans like a humanoid robot. Biped walking using the fuzzy logic controller was verified by the performance of control for the horizontal surface and stair climbing. Fuzzy stabilization of tuning was confirmed by the experiment in a biped robot. Trajectories generator and effectiveness of the method are confirmed by the simulation using the ZMP fuzzy logic controller. The approach of the ZMP method for gait optimization also uses fuzzy logic. 
Li et al. [22] designed the walking upstairs method using an accelerometer sensor and force sensor. Posture analysis of the torso and leg locomotion was discussed. Motion planning is built based on ZMP and feedback sensor for stability.

\section{ORIGINALITY}

In this section, the original research has developed the kids size humanoid robot with 33 Dof namely KMEI humanoid robot. The robot has 33 DoFs in whole bodies, 3 DoFs in head, 6 DoFs in the body, 6 DOFs in the right hand, 6 DoFs in the left hand, and the last 6 DoFs in each leg.

The 3D model of the humanoid robot is shown in Figure 1. The uniqueness of the KMEI humanoid robot is that the robot has a complex joint in the body. The purpose of the complex joint is to make a flexible movement during walking on stairs. The aim focus of this research is on the lower limb of the KMEI humanoid robot. Kinematic and dynamic modeling are used to solve the problem. The simulation of walking on stairs is done using Matlab to verify the results. We also discussed the basic idea of the proposed approach and the reasons for the choice of the approach. KMEI humanoid robot is developed using fuzzy logic control during walking down the stairs.

\section{SYSTEM DESIGN}

The design of the leg joints is connected by serial communication and by distributed control system. The control for several motors and sensors is also placed according to the function and the placement. The whole sensor communication systems are accessed by a microcontroller with serial communication. The main microcontroller of the robot gives the movement commands and calculates every movement in the robot joint using the best kinematics for the moving process. It also transmits all motors in the robot body in real-time. The main control here uses equal or higher computing's rate than the motor's rate so that there is no delay when doing the control in real-time. The humanoid robot itself consists of motion planning and posture control to balance the robot's motion. To achieve the task of climbing a ladder like humans, some things must be prioritized in this task. Based on the control of each robot joint, calculating the swing of the foot when going to step and to move on the surface of the next ladder is very important to prevent collision with the ladder due to calculation errors. Angular and mechanical speed will be very important for this mission to be successful. Planning for this pattern has been calculated based on the identified stair specification factors. The process developed the humanoid robots including $33 \mathrm{DoF}$. The used stair is $0.04 \mathrm{~m}$ tall, $0.6 \mathrm{~cm}$ of wide, and $0.15 \mathrm{~cm}$ of length. Locomotion steps upstairs are a complex task for a humanoid robot. Stability gait is a common factor for balancing during walking in the stairway. The design of stairs reflects the real stair having two steps up and two-steps down. This stair fits for a humanoid robot with notable space to place its feet on the step. KMEI humanoid robot has sensory devices including a pressure 
sensor, distance sensor, gyroscope, and accelerometer. It is used to measure the real joint angles in each joint. IMU measurement including gyroscope and accelerometer is located in the body of the robot to measure slope with the relative gravitational direction.

\subsection{Mechanical Design of KMEI Humanoid Robot}

The mechanical design of the robot is shown in Figure 1. KMEI humanoid robot has $33 \mathrm{DoF}$ (degree of freedom). The configuration of $33 \mathrm{DoF}$ is 12 degrees of freedom on the left and right leg, 6 degrees of freedom on the waist, 3 degrees of freedom on the head, 12 degrees of freedom on the left and right arm. KMEI's humanoid robot has $60 \mathrm{~cm}$ height and $4 \mathrm{~kg}$ weight.
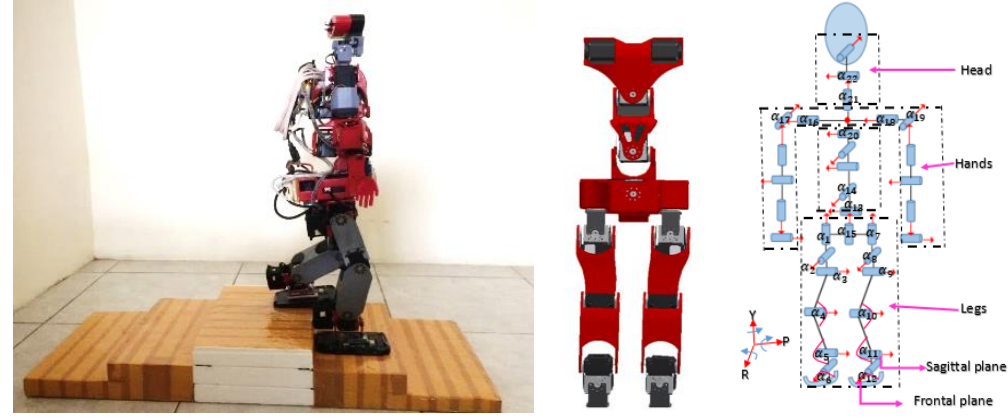

Figure 1. Posture of KMEI humanoid robot during descending stair

Figure 1 shows the mechanical design of the KMEI humanoid robot and the stair. In mechanical, the mechanic is used to derive kinematics and dynamics models from branched kinematics circuits that are connected between links and links. The structure has 33 DoF with the presence of sensors as the feedback controller. The sensors consist of a balance sensor (IMU), a distance sensor, and a pressure sensor located on each robot's leg. These sensors are then combined by using a combination of fuzzy control for sensor feedback. Robot's movement has two phases namely the SSP phase (single support phase) where the phase is one foot into the support and DSP phase (double support phase) where the phase is two feet on the plane surface.

\subsection{Dynamic Modelling of KMEI Humanoid Robot}

To acquire a dynamic system of the humanoid robot, we use the simplification of the seven-links humanoid robot using Lagrange's method [23], process of developing a dynamic model for KMEI humanoid robot. Lagrange equation is the simple one of derivative equation from position and velocity. The generalized coordinates describe the motion of bipedal obtained as:

$$
q_{18 \times 1}=\left[q_{h} q_{l r} q_{n}\right]^{T}
$$

The motion of equation $1 \mathrm{qh}$ is hip motion regarding the base coordinate system (translational and rotational), $\mathrm{q}_{\mathrm{lr}}$ and $\mathrm{q}_{11}$ are relative angles of each leg's joint. The modeling of humanoid robbot has a purpose which can be 
assumed to apply the constraints of the Single Support Phase (SSP) or Double Support Phase (DSP). Moreover, force and moment can substitute the constrains in each phase of the dynamical model for a bipedal robot, shown as:

$$
\mathrm{D}(\mathrm{q}) \ddot{q}+\mathrm{H}(\mathrm{q}, \dot{\mathrm{q}}) \dot{\mathrm{q}}+\mathrm{G}(\mathrm{q}, \dot{\mathrm{q}})=\mathrm{B}
$$

In the equation, $\mathrm{q}$ is $\mathrm{n}$-dimensional vector which describes the joint position of the robot, $\mathrm{B}$ is the vector of input torques, $\mathrm{G}$ is the vector of gravity. Where $\mathrm{D}$ is the $7 \mathrm{x} 7$ inertia matrix (which is symmetric positive definite). $\mathrm{H}$ is 2 vectors of centripetal and Coriolis torques ( $\mathrm{H} 7 \mathrm{x} 7 \mathrm{matrix}$ ) and $\mathrm{G}$ is 2 vectors of gravitational torques. The input of actuators is calculated from the feedback control to perform the desired tasks. The measured system state is also given as vector $\mathrm{q}$ of the joint angles and joint velocities.
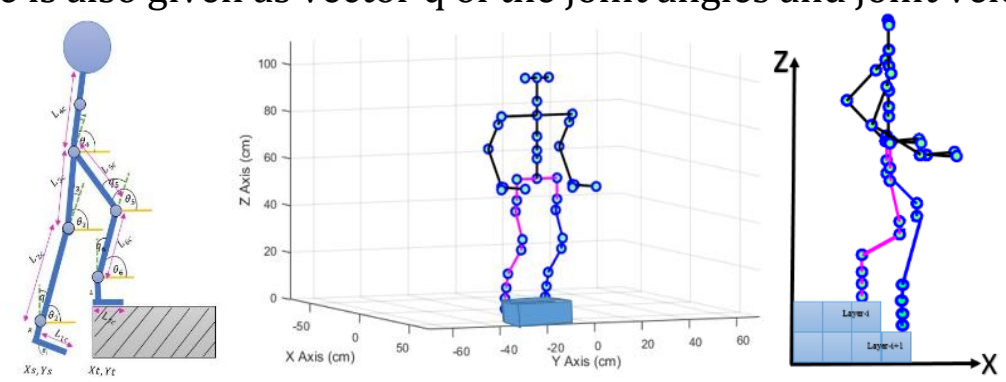

Figure 2. 3D simulation of humanoid robot for descending stair

During descending stair, stage-i is the reference of supported foot on stage $\left(X_{s}, Y_{s}\right)$ and swing foot in stage- $i+1$ for the continuous descending step. The point of the landing foot is the reference foot on the end of the stairs $\left(\mathrm{X}_{\mathrm{t}}\right.$, $\mathrm{Y}_{\mathrm{t}}$ ) as shown in Figure 2. Integrated all of the sensors is used to reduce the impact by the X-axis motion of the foot position and the ground indeed in the feedback controller. Constraints of the motion are caused by the boundary of the robot's dynamic model including the flexible of the robot's mechanics.

\subsection{Double Inverted Pendulum Approach}
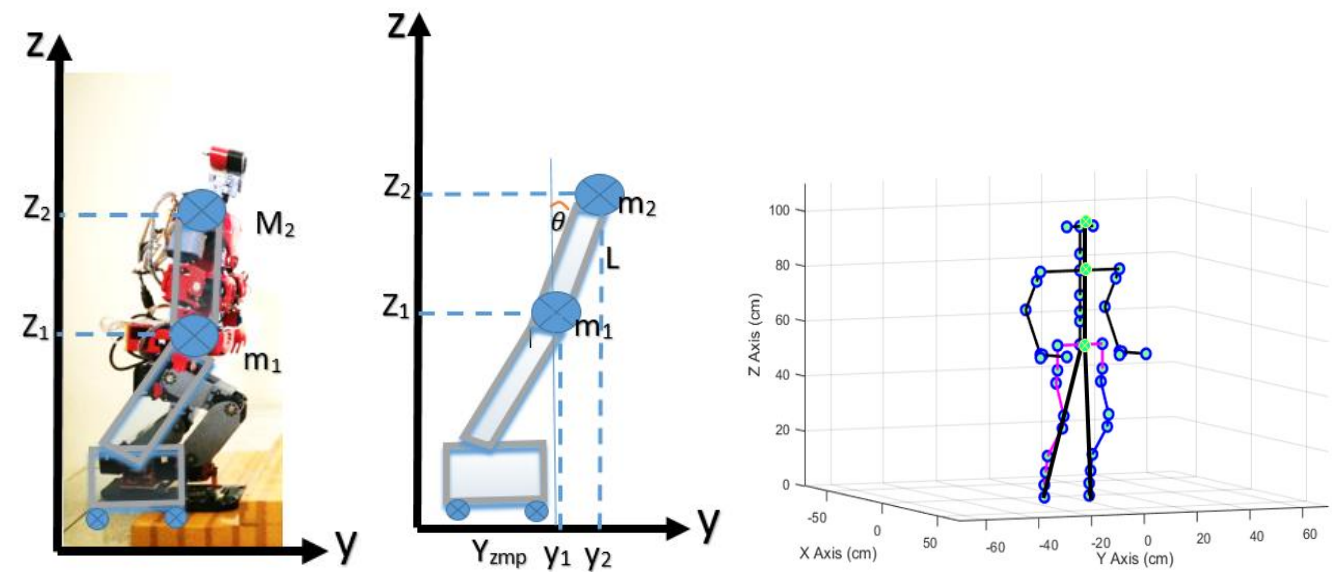

Figure 3. Doube Inverted Pendulum 
The description of triple pendulum approach is divided into three parts, the first part is the two legs, the second part is the body and the third part is head [22]. Each part is illustrated by the one point of mass as shown in Figure 3. The formula for moments points ZMP is obtained:

It represents the body of the humanoid robot based on sagittal motion. Which $m_{1}$ represents the lower body of the center of mass, $m_{2}$ represents the body of the center mass.

$$
m_{1} g\left(x_{1}-x_{\text {smp }}\right)+m_{2} g\left(x_{2}-x_{\text {amp }}\right)=m_{1} z_{1} \ddot{x}_{1}+m_{2} z_{2} \ddot{x}_{2}
$$

Where $\theta$ and $\mathrm{L}$ are the angle and length of the second link respectively, $x 2=x 1+L \sin \theta, z 2=z 1+L \cos \theta$. Substituting these expressions into equation 3 , Where $l$ is the length of the link. The angled joint of the body is $\theta$.

$$
m_{1} g\left(x_{1}-x_{\text {smp }}\right)+m_{2} g\left(x_{1}+L \sin \theta-x_{\text {smp }}\right)=m_{1} z_{1} \ddot{x}_{1}+m_{2}\left(x_{1}+L \cos \theta\right) \ddot{x}_{2}
$$

Based on the constrain that $\theta$ with small deviation $(\theta \approx 0 o)$, we can linearize equation 4 , with $x 2=x 1+L \theta$ and $z 2=z 1+L$. The ZMP equation is derived as

$$
x_{\text {amp }}=x_{1}-\frac{m_{1} z_{1}+m_{2}\left(z_{1}+L\right)}{\left(m_{2}+m_{1}\right) g} \ddot{x}+\frac{m_{2} L}{\left(m_{2}+m_{1}\right)} \theta-\frac{m_{2} L\left(z_{1}+L\right)}{\left(m_{2}+m_{1}\right)} \ddot{\theta}
$$

which can be arranged in matrix form

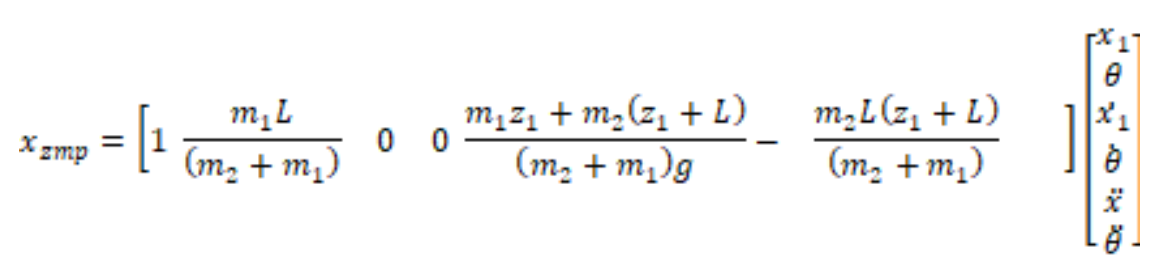

To convert the equation 6 into a standard state-space form, we introduce a

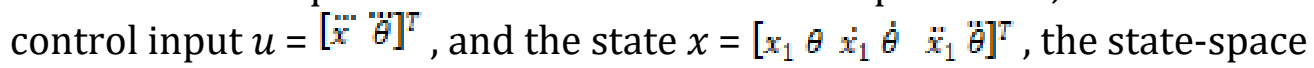
model of the double inverted pendulum can be written as

$$
\left[\begin{array}{c}
\dot{x}_{1} \\
\dot{\theta} \\
\dot{x} \\
\dot{x}_{1} \\
\dot{\theta} \\
\ddot{x} \\
\ddot{\theta}
\end{array}\right]=\left[\begin{array}{llllll}
0 & 0 & 1 & 0 & 0 & 0 \\
0 & 0 & 0 & 1 & 0 & 0 \\
0 & 0 & 0 & 0 & 1 & 0 \\
0 & 0 & 0 & 0 & 0 & 1 \\
0 & 0 & 0 & 0 & 0 & 0 \\
0 & 0 & 0 & 0 & 0 & 0
\end{array}\right]\left[\begin{array}{c}
x_{1} \\
\theta \\
\dot{x}_{1} \\
\dot{\theta} \\
\ddot{x} \\
\ddot{\theta}
\end{array}\right]+\left[\begin{array}{ll}
0 & 0 \\
0 & 0 \\
0 & 0 \\
0 & 0 \\
1 & 0 \\
0 & 1
\end{array}\right]\left[\begin{array}{l}
\ddot{x} \\
z \\
\ddot{\theta}
\end{array}\right]
$$




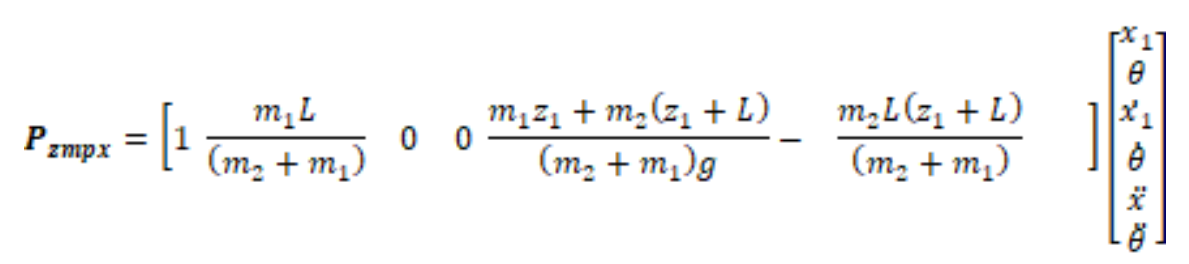

The determinant of the double mass IPM model is

$$
\operatorname{det}=\left[\begin{array}{lll}
S I & -A & -B \\
& C & D
\end{array}\right]
$$

and the transfer function for the double mass IPM is

$G(s)=C(s I-A)^{-1} B=\left[1-\frac{\frac{m_{1} z_{1}+m_{2}\left(z_{1}+L\right)}{\left(m_{2}+m_{1}\right) g} s^{2}}{s^{2}}+\frac{\frac{m_{2} L}{\left(m_{2}+m_{1}\right)}-\frac{m_{2} L\left(z_{1}+L\right)}{\left(m_{2}+m_{1}\right)} s^{2}}{s^{2}}\right]$

Equation 7 and 8 will be used in the gait trajectory generation and further discussion.

\subsection{Ankle Trajectories Generation}

Locomotion of humanoid robot is located on legs especially in ankle joint. During walking on stairs, it has some factors: 1) overstep length stair; 2) swing foot during descending stairs to edge stair which has no adequate slips; 3 ) when the feet landing on the ground using low speed or zero speed [24]. Accordingly, the ankle trajectory can be generated by a cubic polynomial equation 9 . An additional value $a_{n+1}$ is represented by the ground constraints and single-cycle in-ground constraints on the x-direction.

$$
f(x)=a_{0}+a_{1} x+a_{2} x^{2}+\cdots+a_{n+1} x^{n+1}
$$

\subsection{Hip Trajectories Generation}

In this section, the hip trajectory can be generated by the cyclic gait motion. Design and model of hip trajectory are based on the lower model triple inverted pendulum approach [25], one important of walking locomotion on biped robot, one of the hip on a humanoid robot for a base. To generate hip trajectory, there are many parameters affecting walking stability.

$$
\left(L_{y}+L_{1}\right)^{2} \geq x_{\text {hip }}^{2}+z_{\text {hip }}^{2}
$$

Where $L_{y}$ and $L_{\rrbracket}$ are the length of the leg for thigh and shank. To avoid collision with the stair, the support by hip joint should be relatively small position into the stairs, and the constraint that following can be satisfied: 


$$
z_{\text {hip }}-H-x_{\text {hip }} \cdot \tan \frac{s_{d}}{S s} \leq 0
$$

Where $z_{\text {hip }}$ and $x_{\text {hip }}$ are the coordinate vector of the hip. $s_{d}$ is the deep stairs and Ss is step length of stairs. The position of constraints during walking on stairs by the polynomial the hip joint is shown as:

$$
x_{\text {hip }}(t)=\left\{\begin{array}{rl}
x_{h g^{v}} & t=0 \\
x_{\text {hes }} & t=T_{p} \\
x_{h s}+S_{g s} & t=T_{g}
\end{array}\right.
$$

Where $T_{g}$ is the time step and $T_{p}$ is the period. The specification of hip trajectory during walking on stairs with the movement line parallel with the descending stair can be expressed as:

$$
z_{\text {hip }}(t)=H+x_{\text {hip }} \cdot \tan \frac{S_{s}}{S_{d}}
$$

\subsection{Fuzzy Logic Control and Sensory Feedback}

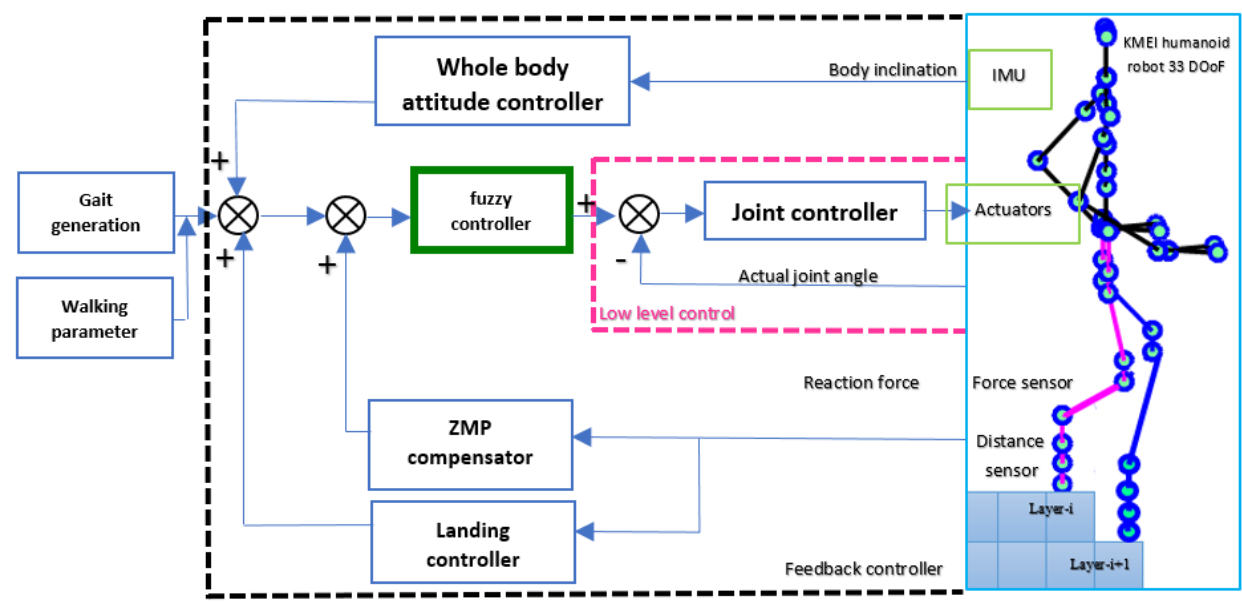

Figure 4. Control strategy system and feedback controller

Stability contraints during walking up the stair on several parts including hp and ankle. The use of several sensors on this robot is to compensate the attitude of the robot when it loses balance and falls in critical conditions due to misaligned environmental conditions. So that the information on the sensor is modified when the robot goes up the stairs. Response of ZMP compensator depend on value sensory thatThe controller uses fuzzy for dynamic balancing of walking. The main method is to keep the balance during walking on the stairs. Balancing system can be improved by the walking on stairs. In Figure 4, the process controller of body inclination can be divided as follows: 1) Which leg is the support during walking on single support phase by the value of center of mass based on force sensors. 
This condition of single support phase is an effect of body attitude. 2) The value of body attitude becomes the input control $\theta$ and $\theta^{\prime}$ for the previous posture. The body's angle for feedback using double inverted pendulum can be represented as follows:

$\theta=$ the angle of actual posture - the angle of desired posture

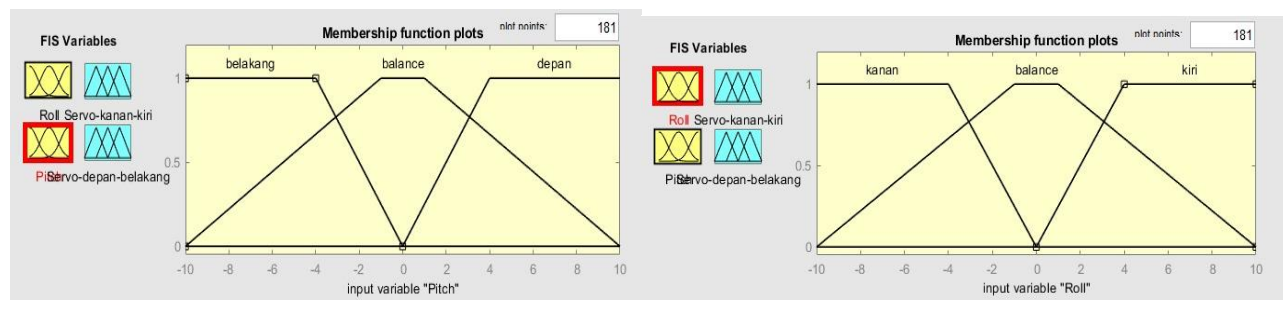

Figure 5. Membership function of angle

Table 1. Rule base of fuzzy

\begin{tabular}{|c|c|c|c|}
\hline Roll $^{\text {Pitch }}$ & N & B & P \\
\hline N & S & L & S \\
\hline B & L & X & L \\
\hline P & S & L & S \\
\hline
\end{tabular}

The value of the input is divided into three partitions. $\mathrm{N}$ (negative), $\mathrm{B}$ (balance), $\mathrm{P}$ (positif). The interference rule is shown in Table 1, where the rule can be represent as follows:

Rule 1: if Roll (N) and Pitch (N) then $\theta$ is $\mathrm{S}$

Rule 2: if Roll (N) and Pitch (B) then $\theta_{\text {is } \mathrm{L}}$

Rule 3: if Roll (N) and Pitch (P) then $\theta_{\text {is } S}$

Rule 9: if Roll (P) and Pitch (P) then $\theta$ is S

\section{EXPERIMENT AND ANALYSIS}

\subsection{Simulation Result}

This section is about the simulation of KMEI humanoid robot with 33 DoF for walking on stairs especially descending stair. The speed parameter for walking of descending stairs is set for $0.015 \mathrm{~m} / \mathrm{s}$. The effects of walking down stair of knee joint angle is divided into 7 steps, shown in Figure 6a. When the robot is walking, the position of the rear body moves into front foot. The foot trajectories during walking down stairs are shown in Figure 6b. 


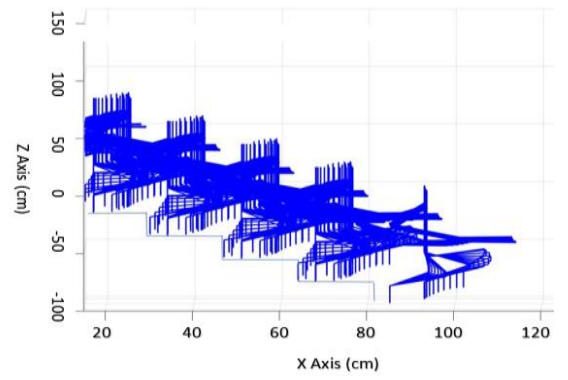

(a) Snapshot descending stair

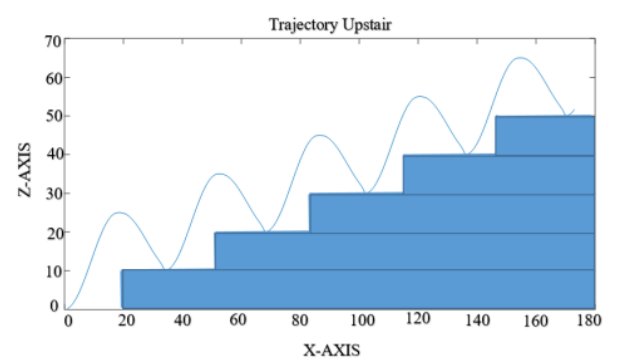

(b) foot trajectory descending stair

Figure 6. Simulation of dynamic walking for descending stair

The simulation of KMEI humanoid robot during descending stair has an affect of the joint body especially of leg on the knee and ankle. The knee joint angle is shown in Figure 7a. The cycle of the walking down stair has a characteristic of periodic behavior. Elevation of knee joint angle almost $25^{0}$ during descending stair. The vertical movement transition by caused swing foot and landing on the ground. The elevation of the torso attitude is shown in Figure $7 \mathrm{~b}$. The position of the single support phase and the center of mass moves forward to the support.

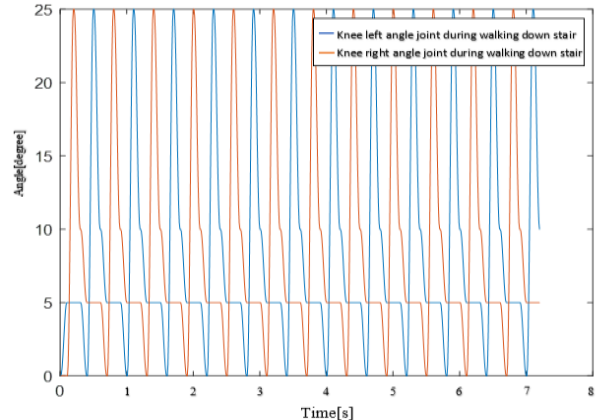

(a) knee joint angle right and left

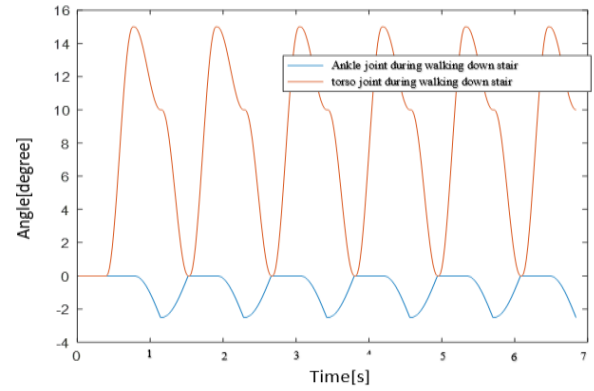

(b) ankle joint angle and torso attitude

Figure 7. Response dynamic walking descending stair

\subsection{Experiments on real KMEI humanoid robot Result}

The realization of KMEI humanoid robot for walking down stair with the hybrid algorithm can be stable and smooth in walking. Step over walking duration is $25 \mathrm{~s}$ for one cycle and the step length is $15 \mathrm{~cm}$. The swing foot on descending stair is more safely because of vertical motion. The Figure 8 shows the realization of KMEI humanoid robot in descending stairs. Walking down stair is divided into 8 steps

a) At the start, from the initial condition in default posture with double support phase and the visualization of the foot sensor center of gravity on the middle robot.

b) Transition idle to movement condition, which skew the body in the one leg and the visualization of pressure sensor can be seen in the single support phase. 
c) Third condition is the swing foot in summit cycle especially it is maximum motion range to landing in the next stair

d) After swing foot is phase landing on stairs, when the foot is located on the stair surface depend on foot sensor. The control strategy is calculated the stair height $H$, the angle joint $\Theta_{d}$. The obstacle in case is stairs acquire the vertical assertion of the ankle.

e) Whole bodies of robot are shifting forward to get center gravity by the controller leg trajectories with the small speed is used to confirm the stability.

f) In the sequel, the swing foot trajectories step over on the next stage by controlled and avoid collision in stair phase. ZMP tracking move into supporting leg through the any phase.

g) At the last, transition of walking from movement onto idle condition, in single support phase. In this step of descending stair is repeated motion to walking in another environment.

h) Final step, default stand on humanoid robot in double support phase. And humanoid robot is beginning to repeat the step. The center gravity is moving in middle of the robot's body.

Foot sensor mechanism can be seen in the visualization. The range of the value of pressure and the distance such as spectral is also shown in the visualization.
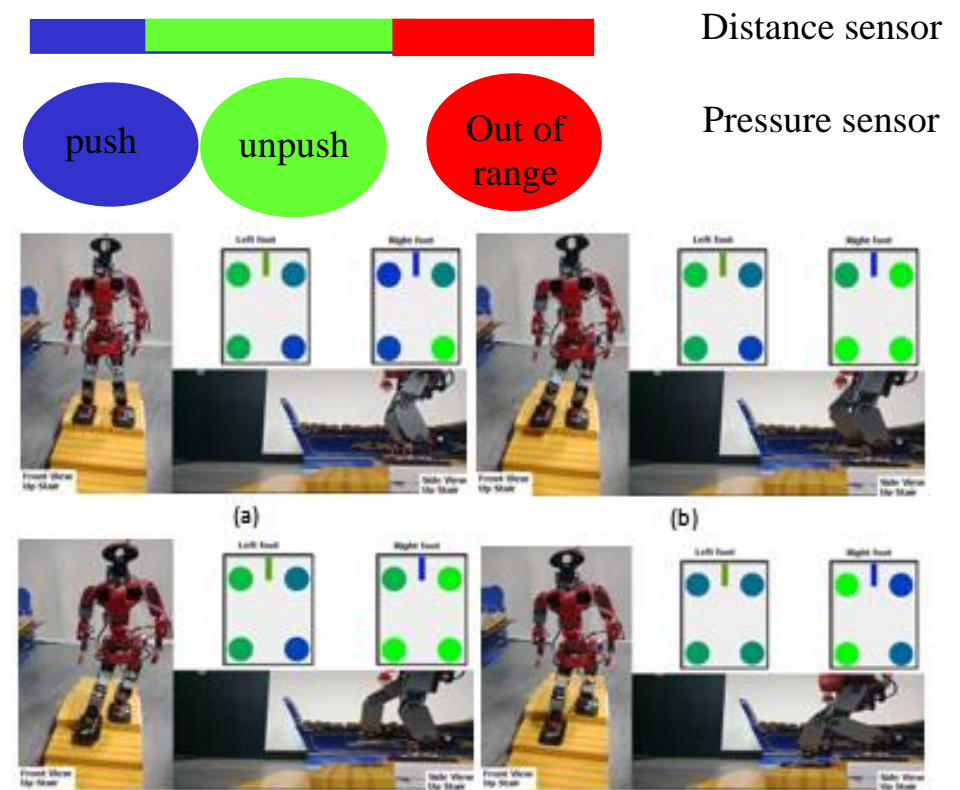

(b)

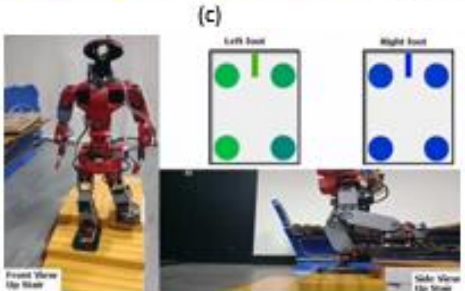

(e)

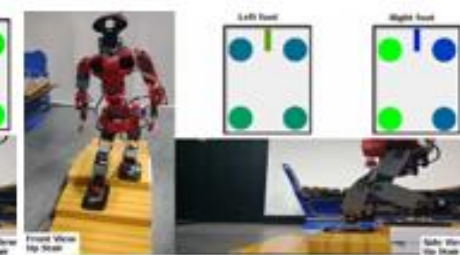

(d)

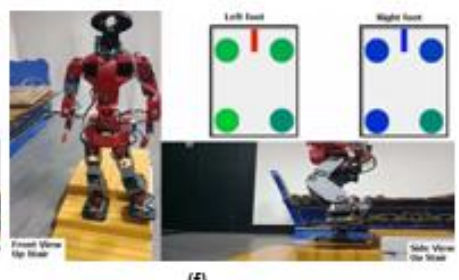

(f) 


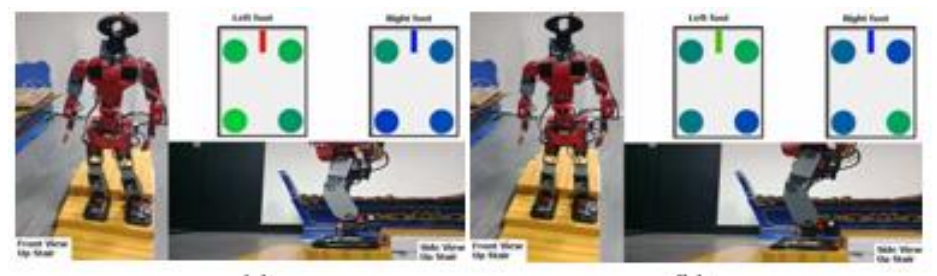

(g)

(h)

Figure 8. Realization of dynamic walking descending stair of KMEI humanoid robot

The experiment of KMEI humanoid robot below uses the hybrid controller and the fusion sensor. The control scheme was successfully applied by the experimental using 33 DoF joint of motion pattern. The stability of walking can be achieved by controlling the single support phase and the double support phase in sagittal plane and frontal plane. Dynamic balance on walking must rely on the fusion of sensors through the ground reaction and the body attitude. Probability of locomotion was considerated by using the mechanics structure and the limitation of the joint angle motion for descending stair.

The performance of humanoid robot can be seen on Figure 9 during walking on stable position. At one time, the condition of ZMP tracking gives out the periodic cycle. In this case, all plans are not allowed, however occur in the region of stability. On the tracking, ZMP is not good on the same time. In Figure 9, it can be seen the disturbance of the vertical force and the errors of desired ZMP and tracking ZMP is small. The result of the tracking ZMP is shown in two directions, $\mathrm{XY}$ direction on top view and $\mathrm{YZ}$ direction on side view.
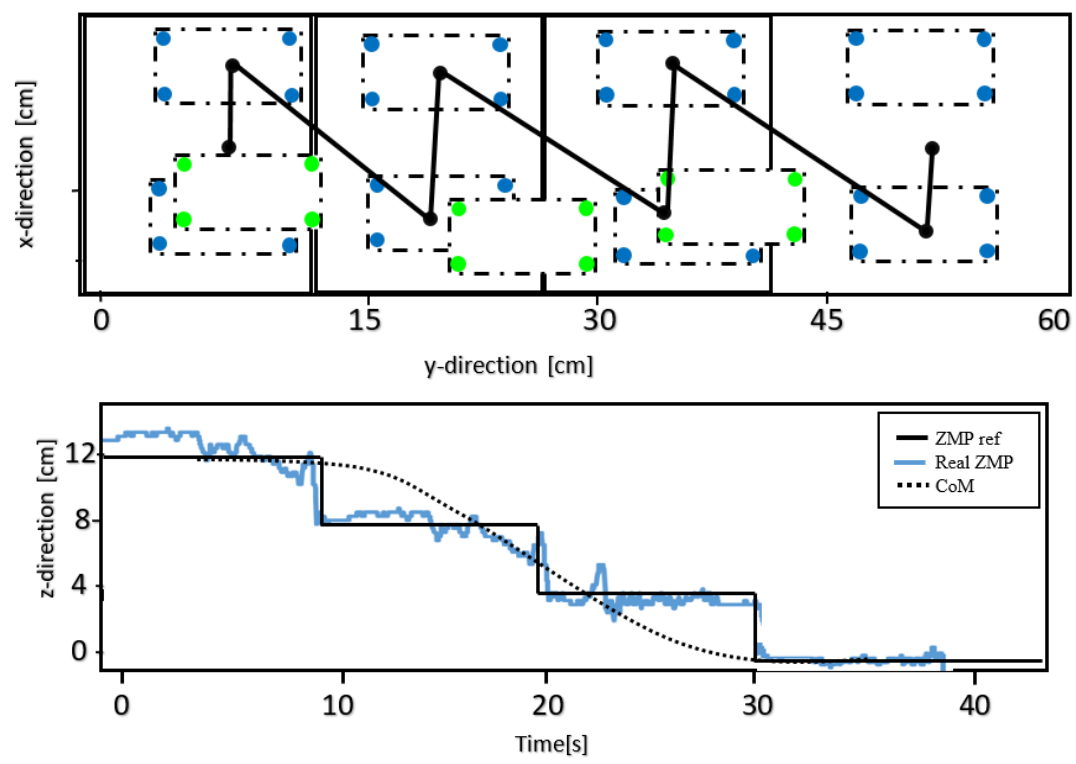


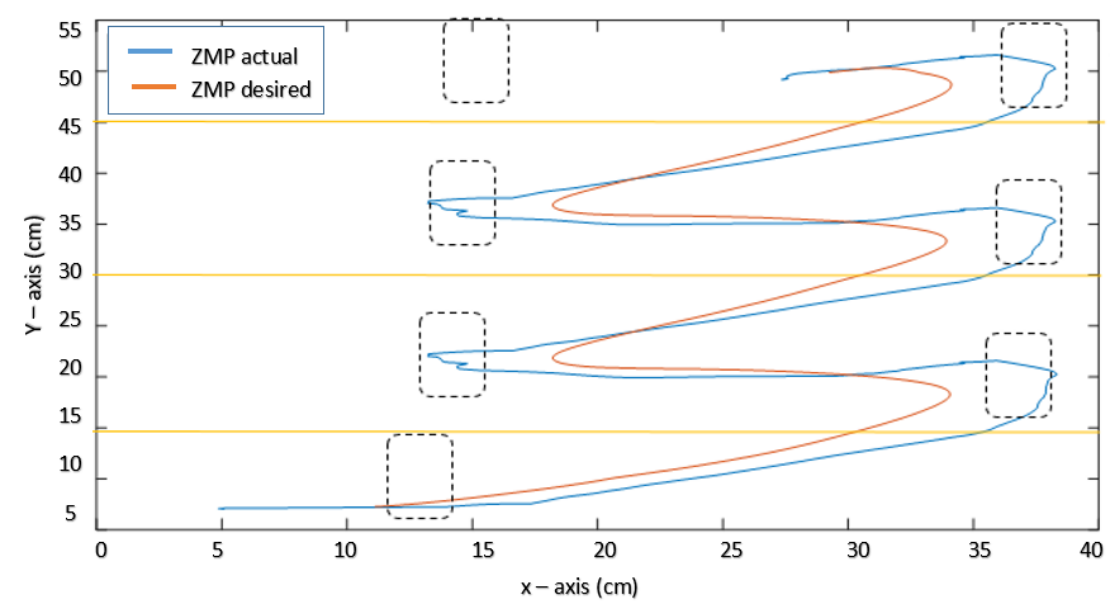

Figure 9. ZMP descending stair

Figure 10 is the variation of different joint angle during the interlude cycle time. The thigh, the ankle and the swing leg have an effect in that interlude. The robot also uses sensory feedback to complete this task. When humanoid robot walking on stair, the condition occurs in single support phase and double support phase. Figure 9 is the response of the joint with sensor feedback. Dynamic walking in the case of descending stair can be seen of top cycle in push position. In case of descending stair by the gravitational effect for swing leg, it can be seen in the thigh.
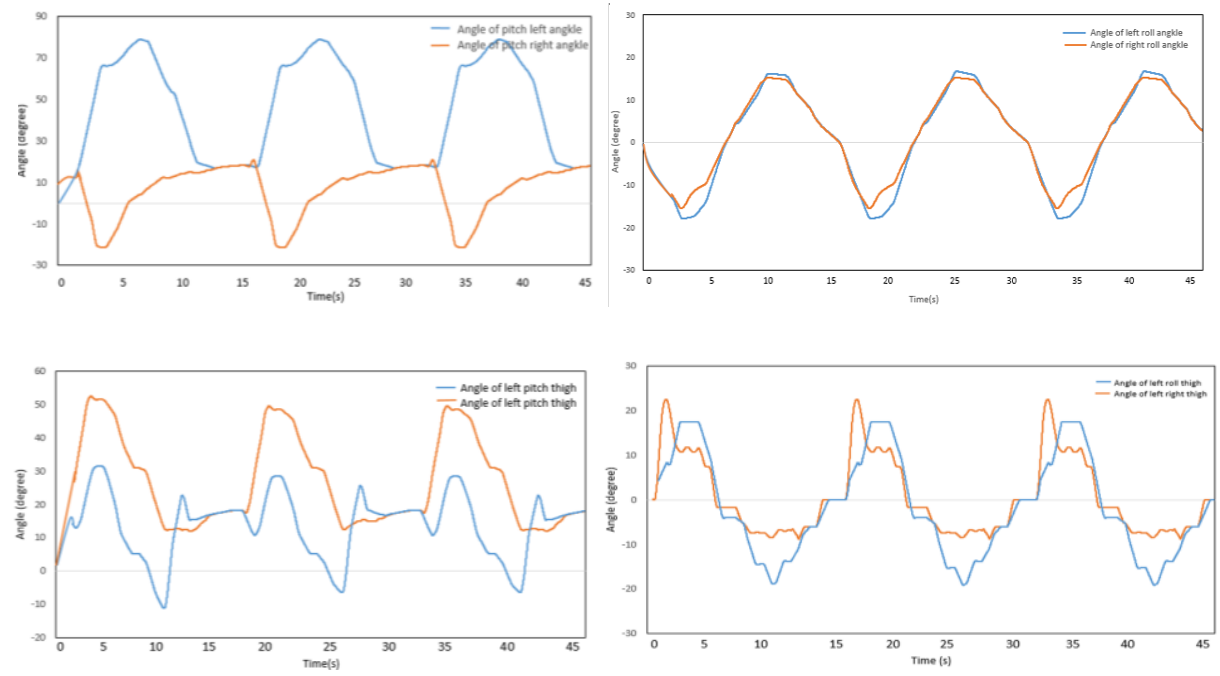

Figure 10. Angle joint for descending stair

\section{CONCLUSION}

This research explains walking on descending stair for KMEI humanoid robot. Fuzzy algorithm is used to control the balance of walking. It relies on the sensor feedback of robot's whole bodies. The setting of parameters for gait walking is limited on joint motion during swing leg and step length. Walking speed for descending stair between implementation and simulation is set differently. Online locomotion is applied during descending stair with inverted 
pendulum approach. Gait generation of humanoid robot is calculated by the parametrical and optimal condition. Thus, The dynamic balance of walking is obtained by the realization on real 33 DoF humanoid robot and verified in the simulation results.

\section{Acknowledgements}

The authors would like to thank all the member of the EEPIS Robotics Research Center (ER2C) laboratory, and Politeknik Elektronika Negeri Surabaya and to Ministry of Research, Technology and Higher Education of the Republic of Indonesia for financial support.

\section{REFERENCES}

[1] S. M. Metev and V. P. Veiko, Laser Assisted Microtechnology, 2nd ed., R. M. Osgood, Jr., Ed. Berlin, Germany: Springer-Verlag, 1998.

[2] Liu, J., Urbann, O.: Bipedal Walking with Dynamic Balance That Involves Three-dimensional Upper Body Motion, Rob. Auton. Syst. 77, 39-54, 2016.

[3] Kobayashi, T., Hasegawa, Y., Sekiyama, K., Aoyama, T., Fukuda, T, Unified Bipedal Gait for Walking and Running by Dynamicsbased Virtual Holonomic Constraint in PDAC, In: Proceedings IEEE Int. Conf. Robot. Autom., pp. 1769-1775, 2016.

[4] Brasseur, C., Sherikov, A., Collette, C., Dimitrov, D., Wieber, P.B, A Robust Linear MPC Approach to Online Generation of 3D Biped Walking Motion, IEEE-RAS Int. Conf. Humanoid Robot. 595-601, 2015.

[5] Shahrokhshahi, Ahmadreza, et al, Optimal Stair Climbing Pattern Generation for Humanoids Using Virtual Slope and Distributed Mass Model, Journal of Intelligent \& Robotic Systems, 94.1 ,43-59, 2019.

[6] Y.-D. Hong and K.-B. Lee, Stable Walking of Humanoid Robots Using Vertical Center of Mass and Foot Motions by an Evolutionary Optimized Central Pattern Generator, International Journal of Advanced Robotic Systems, vol. 13, no. 1, p. 27, 2016.

[7] Liu, Chengju, et al, Active Balance Control of Humanoid Locomotion Based on Foot Position Compensation, Journal of Bionic Engineering, 17.1 , 134-147, 2020.

[8] S. Kuindersma, R. Deits, M. Fallon, A. Valenzuela, $H$. Dai,F.Permenter,T.Koolen,P.Marion,andR.Tedrake, Optimizationbased Locomotion Planning, Estimation, and Control Design for Atlas, Autonomous Robots, vol. 40, no. 3, pp. 429-455, 2016.

[9] J. Carpentier, S. Tonneau, M. Naveau, and O. Stasse, A Versatile and Efficient Pattern Generator for Generalized Legged Locomotion, IEEE International Conference on Robotics and Automation, no. 3, pp. 3555-3561, 2016. 
[10] Morisawa, M. et al, Pattern Generation of Biped Walking Constrained on Parametric Surface. Proc. IEEE Int. Conf. Robot. Autom. 2005, 2405-2410, 2005.

[11] Zhang tong, Research on Walking Control and Motion Planning for Humanoid Robot, Guangzhou: SOUTH CHINA UNIVERSITY OF TECHNOLOGY, 22-23, 2010.

[12] Grizzle J.W., Chevallereau C., Ames A.D., Sinnet R.W, 3D Bipedal Robotic Walking: Models, Feedback Control, and Open Problems. NOLCOS, Bologna, (Italy), 2010.

[13] Zachariah, Sam K., and Thomas Kurian, Hybrid-state Driven Autonomous Control for Planar Bipedal Locomotion Over Randomly Sloped Non-uniform Stairs, Robotics and Autonomous systems 97, 18-39, 2017.

[14] Rajendra, Rega, and Dilip Kumar Pratihar, Analysis of Double Support Phase of Biped Robot and Multi-Objective Optimization Using Genetic Algorithm and Particle Swarm Optimization Algorithm. Sadhana 40.2 , 549-575, 2015.

[15] Ferrari, Paolo, et al, An Integrated Motion Planner/Controller for Humanoid Robots on Uneven Ground. 2019 18th European Control Conference (ECC). IEEE, 2019.

[16] Zhu, Chi, et al, Biped Walking with Variable ZMP, Frictional Constraint, and Inverted Pendulum Model. 2004 IEEE International Conference on Robotics and Biomimetics. IEEE, 2004.

[17] Vundavilli, Pandu Ranga, Sambit Kumar Sahu, and Dilip Kumar Pratihar, Dynamically Balanced Ascending and Descending Gaits of A Twolegged Robot. International Journal of Humanoid Robotics 4.04 , 717 $751,2007$.

[18] J. Englsberger, C. Ott, and A. Albu-Schaffer, Three-dimensional Bipedal Walking Control Based on Divergent Component of Motion, IEEE Transactions on Robotics, vol. 31, no. 2, pp. 355-368, 2015.

[19] Wensing, Patrick M., et al, Optimizing Foot Centers of Pressure Through Force Distribution in a Humanoid Robot. International Journal of Humanoid Robotics 10.03, 1350027, 2013.

[20] Lin J, Chang J, Lyu S M, Wang S W and Lin Y W, Locomotion Control of a Biped Robot for Stair-Climbing by Fuzzy Stabilization Tuning Approach. Proc. of IEEE Multi-Conference on Systems and Control. Yokohama, Japan, September 8-10, pp. 1590-1595, 2010.

[21] Park J H, Fuzzy-Logic Zero-moment-point Trajectory Generation for Reduced Trunk Motions of Biped Robots[J], Fuzzy Sets System, 189203, 2003.

[22] Li, Tzuu-Hseng S., et al, Stair-climbing Control of Humanoid Robot Using Force and Accelerometer Sensors, SICE Annual Conference 2007. IEEE, 2007.

[23] Wang, Pengfei, et al, A Simple Control Algorithm for Controlling Biped Dynamic Walking with Stopping Ability Based on The Footed 
Inverted Pendulum Model, Advances in Mechanical Engineering 8.9 , $1687814016670283,2016$.

[24] Shahrokhshahi, Ahmadreza, et al, Optimal Stair Climbing Pattern Generation for Humanoids Using Virtual Slope and Distributed Mass Model. Journal of Intelligent \& Robotic Systems 94.1 , 43-59, 2019.

[25] Sheng, Bi, et al, Gait Planning of Humanoid Robots Walking on Stairs, FIRA RoboWorld Congress. Springer, Berlin, Heidelberg, 2011. 